\title{
Serotonin and Dopamine Receptor Expression in Solid Tumours Including Rare Cancers
}

\author{
Marloes A. M. Peters ${ }^{1} \cdot$ Coby Meijer $^{1} \cdot$ Rudolf S. N. Fehrmann ${ }^{1} \cdot$ Annemiek M. E. Walenkamp $^{1} \cdot$ Ido P. Kema ${ }^{2}$. \\ Elisabeth G. E. de Vries ${ }^{1} \cdot$ Harry Hollema ${ }^{3}$ Sjoukje F. Oosting ${ }^{1}$
}

Received: 17 December 2018 / Accepted: 27 August 2019 / Published online: 2 September 2019

(C) The Author(s) 2019

\begin{abstract}
In preclinical studies serotonin stimulates and dopamine inhibits tumour growth and angiogenesis. Information regarding serotonin and dopamine receptor (5-HTR and DRD) expression in human cancers is limited. Therefore, we screened a large tumour set for receptor mRNA overexpression using functional genomic mRNA (FGmRNA) profiling, and we analysed protein expression and location of 5-HTR1B, 5-HTR2B, DRD1, and DRD2 with immunohistochemistry in different tumour types. With FGmRNA profiling 11,756 samples representing 43 tumour types were compared to 3,520 normal tissue samples to analyse receptor overexpression. 5-HTR2B overexpression was present in many tumour types, most frequently in uveal melanomas $(56 \%)$. Receptor overexpression in rare cancers included 5-HTR1B in nasopharyngeal carcinoma (17\%), DRD1 in ependymoma $(30 \%)$ and synovial sarcoma (21\%), and DRD2 in astrocytoma (13\%). Immunohistochemistry demonstrated high 5-HTR2B protein expression on melanoma and gastro-intestinal stromal tumour cells and endothelial cells of colon, ovarian, breast, renal and pancreatic tumours. 5-HTR1B expression was predominantly low. High DRD2 protein expression on tumour cells was observed in $48 \%$ of pheochromocytomas, and DRD1 expression ranged from $14 \%$ in melanoma to $57 \%$ in renal cell carcinoma. In conclusion, 5-HTR1B, 5-HTR2B, DRD1, and DRD2 show mRNA overexpression in a broad spectrum of common and rare cancers. 5-HTR2B protein is frequently highly expressed in human cancers, especially on endothelial cells. These findings support further investigation of especially 5HTR2B as a potential treatment target.
\end{abstract}

Keywords Serotonin receptor 1B $\cdot$ Serotonin receptor 2B $\cdot$ Dopamine receptor D2 $\cdot$ Dopamine receptor D1 $\cdot$ Neovascularization Neoplasms

\section{Introduction}

Serotonin and dopamine are biogenic amines, which are produced in the central nervous system and gastrointestinal tract.

Electronic supplementary material The online version of this article (https://doi.org/10.1007/s12253-019-00734-w) contains supplementary material, which is available to authorized users.

Sjoukje F. Oosting

s.oosting@umcg.nl

1 Department of Medical Oncology, University Medical Center Groningen, University of Groningen, Hanzeplein 1, Box 30.001, 9700 RB Groningen, The Netherlands

2 Department of Laboratory Medicine, University Medical Center Groningen, University of Groningen, 9700

RB Groningen, The Netherlands

3 Department of Pathology, University Medical Center Groningen, University of Groningen, 9700 RB Groningen, The Netherlands
Throughout the body, they are transported by platelets. Serotonin and dopamine play a role in vascular tone, gastrointestinal motility, limb movement control and other physiological processes $[1,2]$. Preclinical studies discovered that serotonin and dopamine also influence tumour angiogenesis and tumour growth [3-10].

Angiogenesis is one of the hallmarks of cancer. It is a prerequisite for tumour growth as it secures oxygen and nutrient supply and removal of break-down products from the tumour microenvironment [11]. Serotonin stimulates tumour angiogenesis via activation of serotonin receptor $1 \mathrm{~B}$ (5HTR1B) and serotonin receptor 2B (5-HTR2B) [3-5]. Serotonin can also directly stimulate tumour cell proliferation via various serotonin receptors on tumour cells [10], whereas depletion of serotonin in mice with murine melanoma and lung tumours resulted in slower growth compared with mice having normal serotonin concentrations [5]. Research demonstrated that dopamine inhibits angiogenesis and thereby 
tumour growth in animal models of colon cancer, ovarian cancer, and breast cancer via activation of dopamine receptor D2 (DRD2) [6, 7]. On the opposite, it was shown that the DRD2 pathway is activated in human pancreatic cancer and that growth of pancreatic cancer xenografts was inhibited by DRD2 antagonists in mice [12]. Contradictory results have also been published for dopamine receptor D1 (DRD1), as both inhibition as well as stimulation of tumour growth has been reported upon receptor activation in animal models of ovarian cancer and breast cancer $[6,8,9]$.

Serotonin and dopamine receptor agonists and antagonists are prescribed for the treatment of Parkinson's disease, psychosis, nausea, and migraine [1, 13, 14], but have not been explored for an anti-tumour effect in cancer patients. Information regarding serotonin and dopamine receptor expression in human cancers is limited. Therefore, we screened for 5-HTR1B, 5-HTR2B, DRD1, and DRD2 mRNA overexpression in a large dataset with common and rare tumour types using functional genomic mRNA (FGmRNA) profiling and we determined protein expression and localization of the serotonin and dopamine receptors 5-HTR1B, 5-HTR2B, DRD1, and DRD2 in eight tumour types.

\section{Materials and Methods}

\section{FGmRNA Profiling}

RNA microarray expression data of 11,756 human tumour samples were collected from Gene Expression Omnibus (GEO), a large publicly available data set [15]. From these expression data, FGmRNA profiles were created. Detailed information about FGmRNA profiling was described previously [16]. In short, principal component analysis is used to identify major regulators of the mRNA transcriptome. These so-called transcriptional components are used to correct the mRNA expression data for non-genetic differences, such as physiological and metabolic factors. The expression signal that remains after correction represents variance in mRNA expression due to genetic alterations. This is called the FGmRNA profile.

To determine a threshold for overexpression, FGmRNA profiling of 3,520 normal human tissue samples was performed, and the 97.5th percentile for 5-HTR1B, 5HTR2B, DRD1 and DRD2 was calculated (see Supplementary Table 1 for an overview of non-cancer samples). For all tumour types, the percentage of samples with overexpression was calculated for each of the four receptors. Only tumour types with $>10$ samples available were used for this calculation.

\section{Immunohistochemistry}

\section{Patient Material}

Selection of tumour types for immunohistochemistry was based on results from preclinical studies (colon cancer, ovarian cancer, breast cancer, pancreatic cancer, melanoma) [5-8, 12] or tumour characteristics (high vascularity for renal cell carcinoma, dopamine production for pheochromocytoma, and serotonin-induced proliferation of precursor cells for gastrointestinal stromal tumours (GIST)) [17, 18].

Formalin-fixed paraffin-embedded primary tumour tissues of colon cancer $(n=12)$, ovarian cancer $(n=12)$, breast cancer $(n=12)$, renal cell carcinoma $(n=14)$, and pancreatic cancer $(n=12)$ were used. A minimum of 12 tumours were selected per tumour type, because if $0 / 12$ tumour samples show expression, the chance that it is relevant in $>10 \%$ of patients is low. Furthermore, 3 tissue microarrays (TMA) were used, namely a melanoma TMA containing 36 tumour samples, a pheochromocytoma TMA containing 63 tumour samples, and a GIST TMA containing 76 tumour samples. The TMAs contained 3 cores with a diameter of $0.6 \mathrm{~mm}$ per patient.

Tissue samples used in this study were archival material. According to the Dutch Medical Research Involving Human Subjects act, no approval of the Institutional Review Board was required.

\section{Immunohistochemical Procedure and Analysis}

Formalin-fixed, $4 \mu \mathrm{m}$ thick paraffin-embedded sections and TMAs were deparaffinized and rehydrated. Antigen retrieval was performed using heated citrate buffer ( $\mathrm{pH}$ 6.0) for 5 HTR1B, 5-HTR2B, DRD1, and DRD2 or tris/EDTA buffer ( $\mathrm{pH}$ 9.0) for CD31 for $15 \mathrm{~min}$. For DRD2, slides were blocked with phosphate-buffered saline (PBS; pH 7,4) plus $0.1 \%$ Tween-20 for $20 \mathrm{~min}$ at room temperature. Endogen peroxidase was blocked in all slides with $1 \% \mathrm{H}_{2} \mathrm{O}_{2}$ in PBS for $30 \mathrm{~min}$ at room temperature. For 5-HTR1B, additional avidin/biotin (avidin/biotin blocking kit (SP-2001); Vector Laboratories, Brunschwig Chemie, Amsterdam, The Netherlands) and human serum blocks were performed. The sections were incubated with the primary antibody for $1 \mathrm{~h}$ at room temperature for 5-HTR2B, DRD1, and CD31 or overnight at $4{ }^{\circ} \mathrm{C}$ for 5 -HTR1B and DRD2. Primary antibodies used were mouse monoclonal 5-HTR1B 1:100 (clone 499,325; MAB5858, R\&D systems, Abingdon, United Kingdom), mouse monoclonal 5-HTR2B 1:1000 (clone H11; sc-376,834, Santa Cruz Biotechnology, Bio-Connect, Huissen, The Netherlands), mouse monoclonal DRD2 1:100 (clone B-10, sc-5303, Santa Cruz Biotechnology), rat monoclonal DRD1 1:75 (clone 1-1-F11 s.E6; D2944, Sigma Aldrich, Zwijndrecht, The Netherlands), and mouse 
monoclonal CD31 1:50 (clone JC70A; IR610, DAKO, Glostrup, Denmark). Subsequently, tissue sections were incubated with secondary antibodies (1:100 dilution or in case of 5-HTR1B a 1:300 dilution; all from DAKO). Between steps, slides were washed with PBS or in case of DRD2 with PBS plus $0.1 \%$ Tween-20. Staining was visualized using 3,3'-diaminobenzidine (DAB) and hematoxylin for counterstaining. Positive and negative controls (including immunoglobulin class-matched control sera) were included for each staining. Colon, testis, and prostate tissue served to validate the stainings as positive and negative controls. In addition standard hematoxylin \& eosin (H\&E) stainings were performed to evaluate tissue morphology.

\section{Analysis of Immunohistochemistry}

After immunohistochemical staining, slides were digitally scanned using the NanoZoomer (Hamamatsu Photonics, Shizuoka, Japan) and were scored using accompanied NanoZoomer Digital Pathology (NDP) software. All slides were scored by two independent investigators (M.P. and C.M. or H.H.) and compared to assure a minimal interobserver difference. As an extra control, random slides were also evaluated by a pathologist (H.H.).

Tissue morphology was evaluated using H\&E-stained slides. Staining intensity of the tumour cells and percentage of positive tumour cells was scored. Staining intensity of tumour cells was scored as negative (0), low (1), moderate (2), or high (3). The percentage of positive tumour cells was scored as no positive cells ( 0$), 1-4 \%$ positive cells (1), $5-24 \%$ positive cells (2), $25-49 \%$ positive cells (3), $50-74 \%$ positive cells (4), and $75-100 \%$ positive cells (5). Receptor expression was scored using an immunoreactive score (IRS) to account for the heterogeneous staining in the section slides [19]. The IRS was defined by multiplying the staining intensity (category $0-3$ ) with the percentage of positive tumour cells (category $0-5$ ), creating a range from 0 to 15 . Receptor expression was considered negative if IRS was 0 , low if the IRS was 1 to 5 , and high if the IRS was 6 to 15. For TMAs, at least two evaluable cores had to be present per tumour in order to consider it a representative score.

Receptor expression on tumour-associated blood vessels was only assessed on the whole tissue sections, as blood vessels were only in limited numbers or not at all present in the TMA samples due to their small diameter. Serotonin and dopamine receptor expression was scored by staining intensity (ranging from 0 to 3 ). Receptor expression was considered negative if intensity was 0 , low if intensity was 1 , and high if intensity was 2 or 3 , CD31 staining was performed to confirm localization of tumour vessels.

\section{Results}

\section{Overexpression of Serotonin and Dopamine Receptors Analysed with FGmRNA Profiling}

For the frequency of overexpression of serotonin and dopamine receptors per tumour type, see Table 1.

5-HTR1B was overexpressed in a low percentage of tumour samples per tumour type. The highest percentage was found in nasopharyngeal carcinoma: in 7 of $42(17 \%)$ samples.

Of the four receptors, 5-HTR2B was most frequently overexpressed, especially in uveal melanomas (59 of 106 (56\%) samples). Also in certain brain tumours and sarcomas, and in hepatocellular carcinoma and non-small cell lung cancer, a relatively high proportion of tumour samples showed overexpression of 5-HTR2B: 6 out of 24 astrocytomas (25\%), 15 out of 76 liposarcomas (20\%), 4 out of 26 osteosarcomas $(15 \%), 71$ out of 346 hepatocellular carcinomas $(20 \%)$ and 16 out of 103 small cell lung cancers $(16 \%)$.

Overexpression of DRD1 was most frequently found in ependymoma and synovial sarcoma with 46 out of 156 $(30 \%)$ respectively 7 out of $34(21 \%)$ of the samples.

DRD2 was most frequently overexpressed in adrenal neuroblastoma (24 out of $96(25 \%)$ samples) and astrocytoma (3 out of 24 (13\%) samples).

\section{Serotonin and Dopamine Receptor Expression Analysed with Immunohistochemistry}

Tumour cells predominantly had low or absent 5-HTR1B expression, except for 10 out of 12 colon cancers and 4 out of 12 ovarian cancers showing high 5-HTR1B expression. 5HTR2B was highly expressed on tumour cells of all melanomas and all but 2 GIST. Pheochromocytomas showed either absent or very high expression of 5-HTR2B on tumour cells. In some pheochromocytomas $(n=12)$, also small foci of strongly positive tumour cells were observed within a field of negative tumour cells. DRD1 was highly expressed on tumour cells of approximately $50 \%$ of colon cancers, ovarian cancers, breast cancers, renal cell carcinomas and GIST samples. For melanoma and pheochromocytoma, this was the case in less than $25 \%$ of samples. High DRD2 expression by tumour cells was most frequently observed in pheochromocytomas (30 out of 63 samples), ovarian cancer ( 6 out of 12 samples) and pancreatic cancer (6 out of 12 samples) (Fig. 1).

5-HTR1B expression was predominantly low or absent on endothelial cells, except for 6 out of 12 colon cancers and 4 out of 12 ovarian cancers. 5-HTR2B, on the other hand, was highly expressed on endothelial cells of all tumour types investigated (colon, ovarian, breast, renal, and pancreatic cancer). Expression of DRD1 on endothelial cells varied per 
Table 1 Overexpression of serotonin and dopamine receptors in different tumour types determined with functional genomic mRNA analysis

Percentage overexpression (\%)

Tumor type (N)

\begin{tabular}{llll}
\hline 5-HTR1B & 5-HTR2B
\end{tabular}

Breast cancer

ER-/Her2+ (455)

ER+/Her2- $(1,678)$

6.6

3.5

2.0

1.1

3.4

4.5

1.0

0.6

$\mathrm{ER}+/ \mathrm{Her} 2+(506)$

TNBC (737)

0.7

CNS malignancies

Anaplastic astrocytoma (36)

5.6

Anaplastic oligodendroglioma (26)

Astrocytoma (24)

Ependymoma (156)

Glioblastoma (389)

Medulloblastoma (148)

Meningioma (122)

Oligodendroglioma (23)

Pilocytic astrocytoma (135)

Endocrine malignancies
Adrenal neuroblastoma (96)

Adrenocortical carcinoma (20)

Anaplastic thyroid carcinoma (21)

Papillary thyroid carcinoma (51)

Gastrointestinal malignancies

Colorectal cancer $(2,710)$

Esophageal adenocarcinoma (41)

Esophageal squamous cell carcinoma (56)

Gastric cancer (332)

Hepatocellular carcinoma (364)

Pancreatic cancer (81)

4.2

0.0

0.0

2.0

\section{3}

4.9

0.0

3.9

3.3

0.0

Genitourinary malignancies

Bladder cancer (39)

Chromophobe renal cancer (37)

Clear cell renal cancer (225)

Papillary renal cancer (37)

Prostate cancer (308)

Gynaecological malignancies

Cervical cancer (62)

Ovarian cancer (187)

1.6

0.5

4.9

16.7

1.6

7.8

5.2

10.0

14.3

7.8

4.3

14.6

3.6

12.0

19.5

8.6

\section{7}

0.0

7.6

0.0

5.2

6.5

3.7

0.6

11.9

Lung cancer

Adenocarcinoma (1,019)
2.8

0.0

4.2

29.5

1.3

0.7

0.0

4.3

0.0

2.0

0.0

1.2

2.4 
Table 1 (continued)

Percentage overexpression $(\%)$

\begin{tabular}{|c|c|c|c|c|}
\hline \multirow[b]{2}{*}{ Tumor type $(\mathrm{N})$} & \\
\hline & 5-HTR1B & 5-HTR2B & DRD1 & DRD2 \\
\hline Squamous cell carcinoma (405) & 0.2 & 2.7 & 1.5 & 0.5 \\
\hline Small cell lung cancer (103) & 1.0 & 15.5 & 4.9 & 8.7 \\
\hline \multicolumn{5}{|l|}{ Melanoma } \\
\hline Cutaneous (398) & 3.8 & 9.0 & 0.0 & 1.3 \\
\hline Uveal (106) & 2.8 & 55.7 & 2.8 & 0.9 \\
\hline \multicolumn{5}{|l|}{ Sarcoma } \\
\hline Ewing's sarcoma (26) & 3.8 & 0.0 & 0.0 & 3.8 \\
\hline Leiomyosarcoma (60) & 1.7 & 6.7 & 5.0 & 5.0 \\
\hline Liposarcoma (76) & 3.9 & 19.7 & 1.3 & 1.3 \\
\hline Osteosarcoma (26) & 0.0 & 15.4 & 0.0 & 0.0 \\
\hline Primitive neuroectodermal tumor (22) & 4.5 & 0.0 & 0.0 & 4.5 \\
\hline Synovial sarcoma (34) & 0.0 & 0.0 & 20.6 & 0.0 \\
\hline Undifferentiated sarcoma (95) & 1.1 & 9.5 & 1.1 & 0.0 \\
\hline
\end{tabular}

N; number, 5-HTR1B; serotonin receptor 1B, 5-HTR2B; serotonin receptor 2B, DRD1; dopamine receptor D1, DRD2; dopamine receptor D2, ER; estrogen receptor, Her2; human epidermal growth factor receptor 2, TNBC: triple negative breast cancer, CNS; central nervous system, HNSCC; head and neck squamous cell carcinoma

tumour type. In breast cancer, DRD1 was highly expressed on endothelial cells of 7 out of 12 samples. For the other tumour types, high expression of DRD1 on endothelial cells was only observed in 4 out of 14 renal cell carcinomas and 1 pancreatic cancer sample. DRD2 expression was low or absent on endothelial cells (Fig. 2).

For representative images of serotonin and dopamine receptor staining on ovarian cancer cells and tumour blood vessels, see Fig. 3a and b.
Fig. 1 a Serotonin receptor 1B (5-HTR1B), b serotonin receptor 2B (5-HTR2B), c dopamine receptor D1 (DRD1), $\mathbf{d}$ and dopamine receptor D2 (DRD2) expression on tumour cells of colon cancer $(\mathrm{CC})(n=12)$, ovarian cancer (OC) $(n=12)$, breast cancer $(\mathrm{BC})(n=12)$, renal cell carcinoma $(\mathrm{RCC})(n=14)$, pancreatic cancer $(\mathrm{PC})(n=12)$, melanoma $(\mathrm{M})(n=36)$, pheochromocytoma (PCC) $(n=63)$ and gastrointestinal stromal tumours (GIST) $(n=76)$ as analysed with immunohistochemistry.

Immunoreactive score (IRS) was used to classify receptor expression on tumour cells in three categories: negative (in white), low (in light grey) or high (in dark grey)
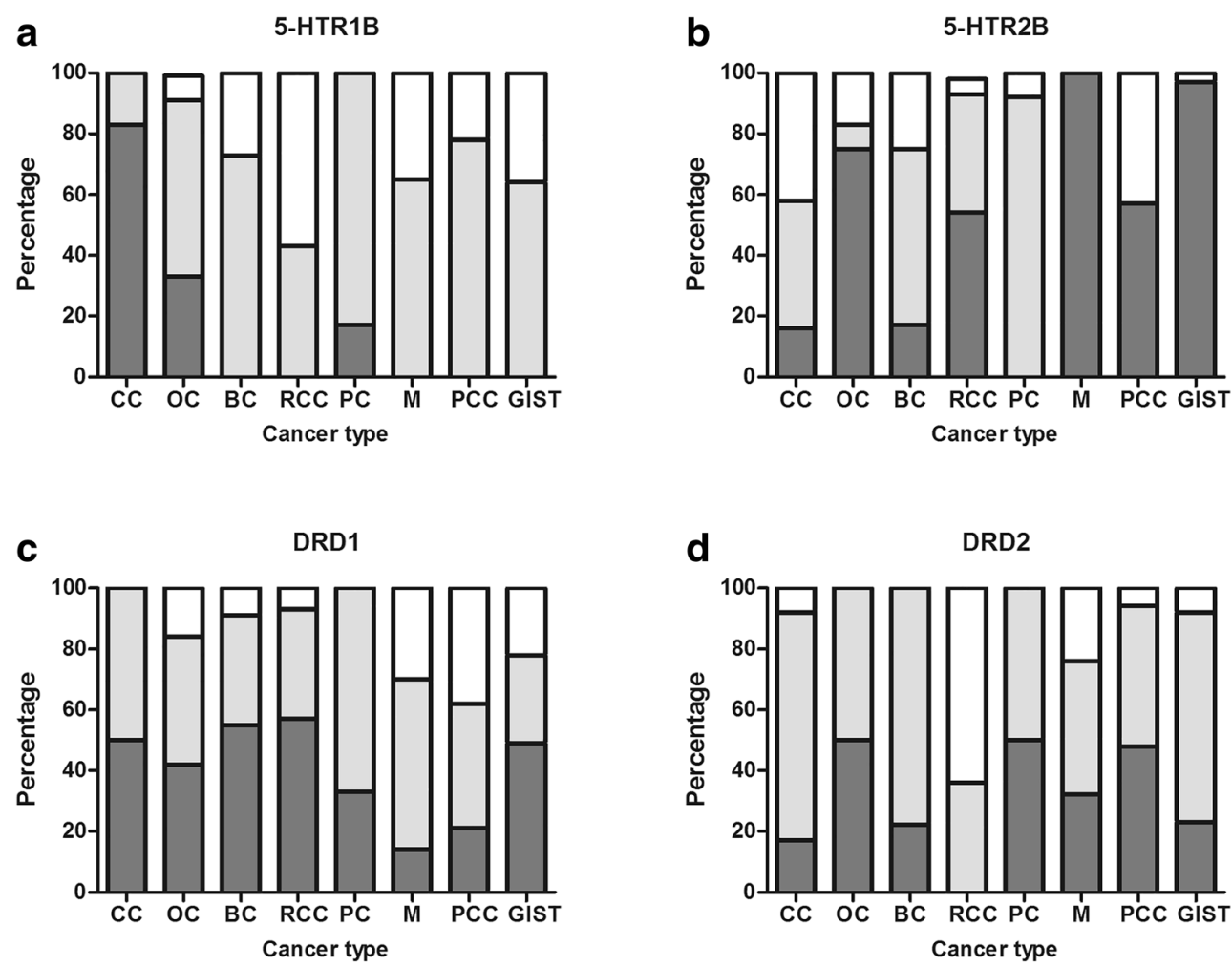

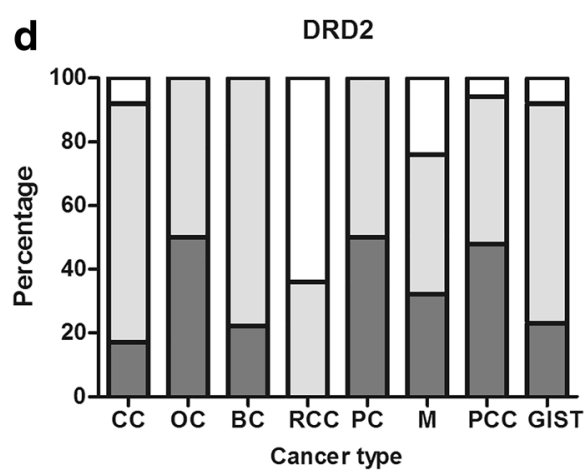


Fig. 2 a Serotonin receptor $1 \mathrm{~B}$ (5-HTR1B), b serotonin receptor 2B (5-HTR2B), c dopamine receptor D1 (DRD1), $\mathbf{d}$ and dopamine receptor D2 (DRD2) expression on endothelial cells of colon cancer (CC) $(n=12)$, ovarian cancer $(\mathrm{OC})(n=12)$, breast cancer $(\mathrm{BC})(n=12)$, renal cell carcinoma (RCC) $(n=14)$, and pancreatic cancer (PC) $(n=12)$ as analysed with immunohistochemistry. Receptor expression was classified by staining intensity. Receptor expression was considered negative if intensity was 0 (white), low if intensity was 1 (in light grey), and high if intensity was 2 or 3 (in dark grey) a

5-HTR1B

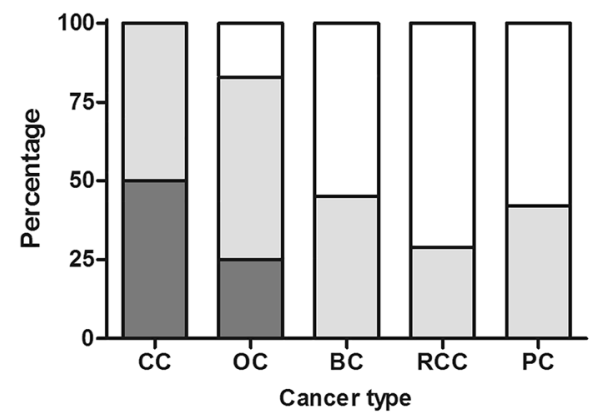

C

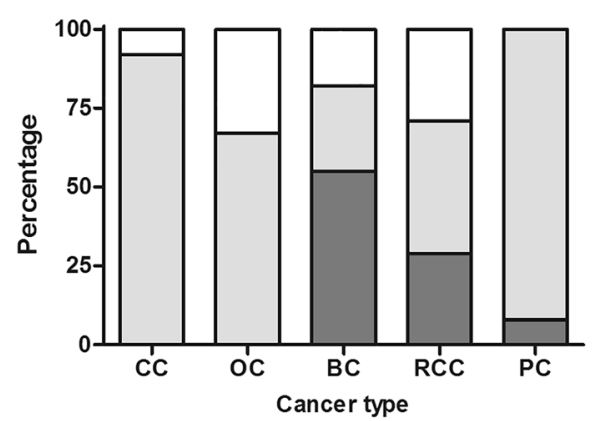

b 5-HTR2B

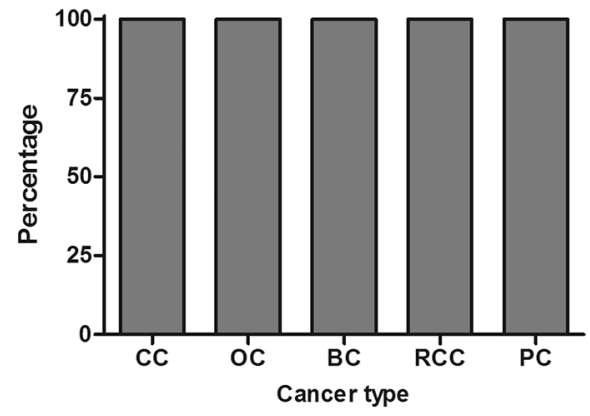

d

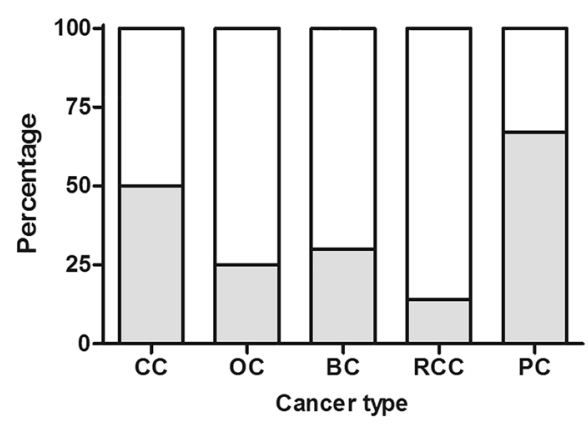

\section{Discussion}

To our knowledge, this is the first study evaluating 5-HTR1B, 5-HTR2B, DRD1, and DRD2 (over)expression in a broad range of tumour types with both FGmRNA profiling and immunohistochemistry. We found that 5-HTR2B is more frequently overexpressed compared to 5-HTR1B, DRD1, and DRD2. All four receptors, however, are expressed across tumour types including rare cancers. 5-HTR2B is highly expressed on tumour cells in all melanomas and on tumour endothelial cells of colon, ovarian, breast, renal, and pancreatic cancer samples.

One of the great advantages of FGmRNA is that it enabled us to screen overexpression of serotonin and dopamine receptors in a large number of samples, leading to interesting findings in rare cancers like brain tumours and sarcomas. FGmRNA profiling identified mRNA overexpression based on a strictly chosen cut-off level, being receptor expression higher than the 97.5th percentile of expression in normal tissue (16). This may have led to the relatively low overexpression percentages found with FGmRNA profiling compared with the number of samples with high expression found with immunohistochemistry.

FGmRNA profiling and immunohistochemistry both demonstrated high 5-HTR2B expression in (uveal) melanoma. Two in vitro studies evaluated the effect of serotonin on melanoma growth with opposite results. Serotonin inhibited proliferation of 5-HTR2B expressing human melanoma cell line
IPC298 but it did not affect B16F0 murine melanoma cell proliferation, in which however, receptor expression was not assessed $[5,20]$. On the other hand, mice with serotonin depletion due to knockout of a serotonin transporter had smaller B16F0 murine melanomas than mice with a functional serotonin transporter and thus unaffected serotonin concentrations in blood [5].

5-HTR2B was highly expressed on endothelial cells of five tumour types evaluated using immunohistochemistry (colon cancer, ovarian cancer, breast cancer, renal cell carcinoma, and pancreatic cancer). This was in concordance with studies from a research group from Malmö, Sweden, which demonstrated 5-HTR2B protein expression by endothelial cells in 29 ovarian cancer and 102 breast cancer samples [21, 22]. In preclinical studies, the effect of 5-HTR2B antagonists on angiogenesis was evaluated: phosphorylation of serotonininduced endothelial nitric oxide synthase (eNOS) was blocked in human umbilical vein endothelial cells (HUVEC) and in a murine lung cancer model, and was associated with decreased tumour microvessel density [5].

Preclinical research demonstrated that dopamine inhibits tumour angiogenesis via activation of DRD2. To our knowledge, this is the first study evaluating DRD2 expression on vasculature of human tumours. Previous studies demonstrated DRD2 protein expression on vessels of mouse ears and HUVEC [6]. In our study however, we observed low or absent DRD2 protein expression on tumour-associated endothelial cells. On tumour cells of 30/63 pheochromocytomas, DRD2 

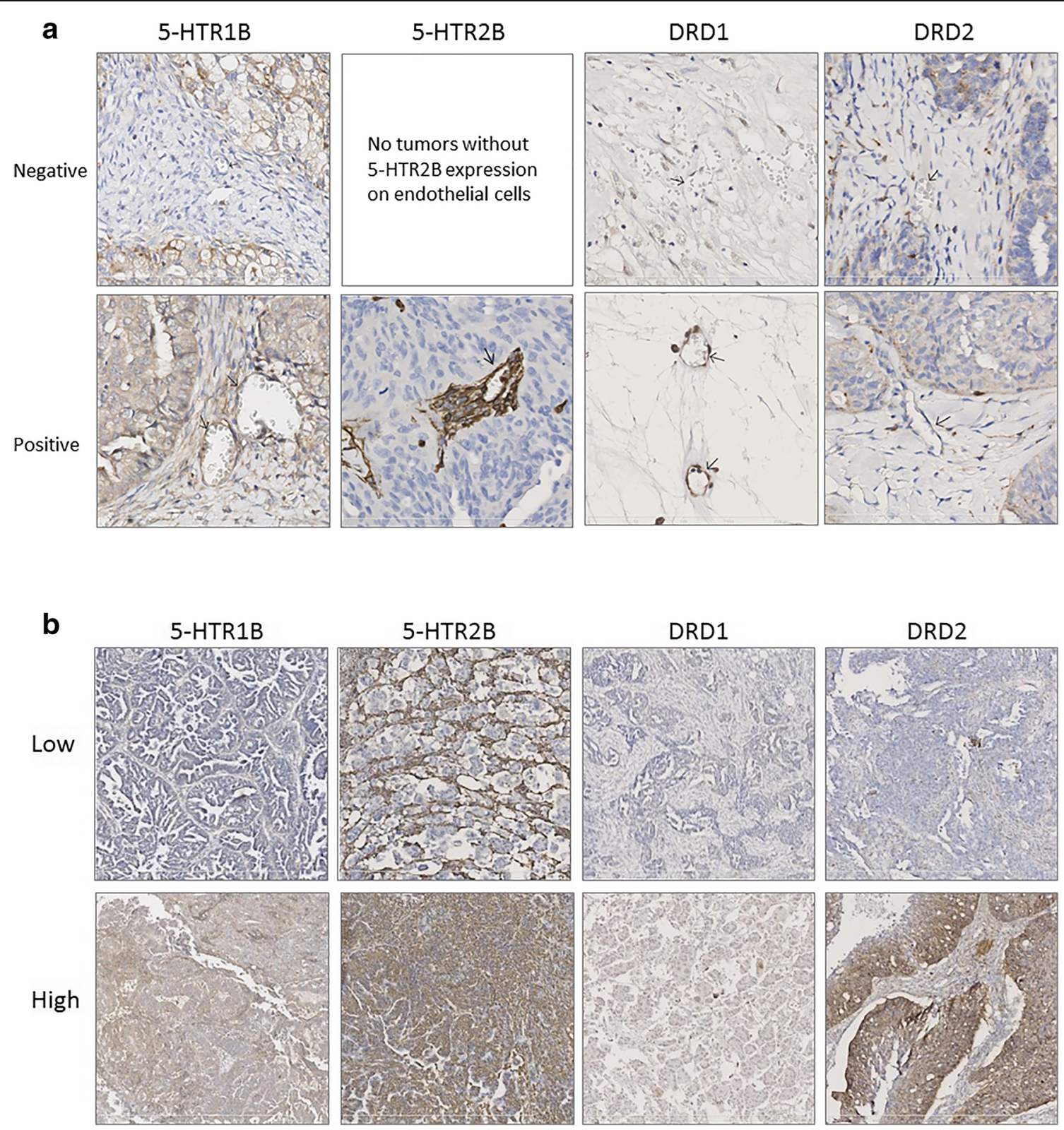

Fig. 3 a Representative images of low and high expression of serotonin receptor 1B (5-HTR1B), serotonin receptor 2B (5-HTR2B), dopamine receptor D1 (DRD1), and dopamine receptor D2 (DRD2) by tumour cells in ovarian cancer (10x magnification). b Representative images of negative and positive (low or high) expression of serotonin receptor 1B (5-

HTR1B), serotonin receptor 2B (5-HTR2B), dopamine receptor D1 (DRD1), and dopamine receptor D2 (DRD2) on blood vessels in ovarian cancer (40x magnification). Arrowheads indicate blood vessels that express the receptor of interest

protein was highly expressed. This was in concordance with smaller studies in 10 respectively 39 pheochromocytomas [23, 24]. Interestingly, a phase I study with the DRD2 antagonist ONC201, demonstrated some clinical benefit in five endometrial and prostate cancer patients from a group of 27 advanced cancer patients [25]. However, a phase II study with ONC201 in 17 recurrent glioblastoma patients was closed after interim analysis since the target of a six month progression-free survival in 30\% of patients was not reached [26]. In both studies, DRD2 expression was not evaluated. If the patient population could have been enriched based on target expression remains therefore unclear. Pituitary adenoma is a tumour type known to express DRD2, and the DRD2 agonist bromocriptine is already part of the standard treatment regimen in prolactinproducing adenomas $[1,27]$. Dependent on tumour type and DRD2 expression on tumour cells, treatment with a DRD2 agonist or antagonist may have anti-tumour activity. For antiangiogenic treatment however, the significance of DRD2 agonists seems limited based on our results regarding expression of this receptor on tumour-associated blood vessels.

In conclusion, serotonin and dopamine receptors are differentially (over)expressed in various tumour types by tumour and 
endothelial cells. Of these, 5-HTR2B is expressed most frequently. This study demonstrates that selection of patients with tumours of different backgrounds but with similar receptor expression profiles is possible. This could offer interesting future possibilities for basket studies. Basket studies include different tumour types and select patients with the same tumour characteristic for targeted treatment [28]. This allows to study new treatment modalities in rare tumours, such as brain tumours and sarcomas, for which there are currently limited treatment options.

Acknowledgements We would like to thank W. Boersma-van Ek and T. van der Sluis for technical assistance.

Author Contributions All authors contributed to the conception and design of the study. MP carried out the experiments. MP, CM, and $\mathrm{HH}$ scored the immunohistochemistry data. RF designed the FGmRNA profiling. All authors contributed to interpretation of the data, and were involved in writing the paper and had final approval of the submitted and published versions.

Funding Information M.A.M. Peters received a Junior Scientific Master class/Ubbo Emmius Foundation Talent Grant of the Van der MeerBoerema Foundation and a University of Groningen/Junior Scientific Master class grant for $\mathrm{PhD}$ students.

\section{Compliance with Ethical Standards}

Conflict of Interest The authors declare that they have no conflict of interest.

Open Access This article is distributed under the terms of the Creative Commons Attribution 4.0 International License (http:// creativecommons.org/licenses/by/4.0/), which permits unrestricted use, distribution, and reproduction in any medium, provided you give appropriate credit to the original author(s) and the source, provide a link to the Creative Commons license, and indicate if changes were made.

\section{References}

1. Beaulieu JM, Gainetdinov RR (2011) The physiology, signaling, and pharmacology of dopamine receptors. Pharmacol Rev 63:182217

2. Jonnakuty C, Gragnoli C (2008) What do we know about serotonin? J Cell Physiol 217:301-306

3. Qin L, Zhao D, Xu J, Ren X, Terwilliger EF, Parangi S, Lawler J, Dvorak HF, Zeng H (2013) The vascular permeabilizing factors histamine and serotonin induce angiogenesis through TR3/Nur77 and subsequently truncate it through thrombospondin-1. Blood $121: 2154-2164$

4. Zamani A, Qu Z (2012) Serotonin activates angiogenic phosphorylation signaling in human endothelial cells. FEBS Lett 586:2360 2365

5. Asada M, Ebihara S, Yamanda S, Niu K, Okazaki T, Sora I, Arai H (2009) Depletion of serotonin and selective inhibition of 2B receptor suppressed tumor angiogenesis by inhibiting endothelial nitric oxide synthase and extracellular signal-regulated kinase $1 / 2$ phosphorylation. Neoplasia 11:408-417

6. Basu S, Nagy JA, Pal S, Vasile E, Eckelhoefer IA, Susan Bliss V, Manseau EJ, Dasgupta PS, Dvorak HF, Mukhopadhyay D (2001)
The neurotransmitter dopamine inhibits angiogenesis induced by vascular permeability factor/vascular endothelial growth factor. Nat Med 7:569-574

7. Sarkar C, Chakroborty D, Chowdhury UR, Dasgupta PS, Basu S (2008) Dopamine increases the efficacy of anticancer drugs in breast and colon cancer preclinical models. Clin Cancer Res 14: 2502-2510

8. Moreno-Smith M, Lee SJ, Lu C, Nagaraja AS, He G, Rupaimoole R, Han HD, Jennings NB, Roh JW, Nishimura M, Kang Y, Allen JK, Armaiz GN, Matsuo K, Shahzad MMK, Bottsford-Miller J, Langley RR, Cole SW, Lutgendorf SK, Siddik ZH, Sood AK (2013) Biologic effects of dopamine on tumor vasculature in ovarian carcinoma. Neoplasia 15:502-510

9. Borcherding DS, Tong W, Hugo ER et al (2016) Expression and therapeutic targeting of dopamine receptor-1 (D1R) in breast cancer. Oncogene 35:3103-3113

10. Peters MA, Walenkamp AM, Kema IP, Meijer C, De Vries EG, Oosting SF (2014) Dopamine and serotonin regulate tumor behavior by affecting angiogenesis. Drug Resist Updat 17:96-104

11. Hanahan D, Weinberg RA (2011) Hallmarks of cancer: the next generation. Cell 144:646-674

12. Jandaghi P, Najafabadi HS, Bauer AS, Papadakis AI, Fassan M, Hall A, Monast A, von Knebel Doeberitz M, Neoptolemos JP, Costello E, Greenhalf W, Scarpa A, Sipos B, Auld D, Lathrop M, Park M, Büchler MW, Strobel O, Hackert T, Giese NA, Zogopoulos G, Sangwan V, Huang S, Riazalhosseini Y, Hoheisel JD (2016) Expression of DRD2 is increased in human pancreatic ductal adenocarcinoma and inhibitors slow tumor growth in mice. Gastroenterology 151:1218-1231

13. Jordan K, Gralla R, Jahn F, Molassiotis A (2014) International antiemetic guidelines on chemotherapy induced nausea and vomiting (CINV): content and implementation in daily routine practice. Eur J Pharmacol 722:197-202

14. Peterlin BL, Rapoport AM (2007) Clinical pharmacology of the serotonin receptor agonist, zolmitriptan. Expert Opin Drug Metab Toxicol 3:899-911

15. Barrett T, Wilhite SE, Ledoux P, Evangelista C, Kim IF, Tomashevsky M, Marshall KA, Phillippy KH, Sherman PM, Holko M, Yefanov A, Lee H, Zhang N, Robertson CL, Serova N, Davis S, Soboleva A (2013) NCBI GEO: archive for functional genomics data sets - update. Nucleic Acids Res 41:D991-D995

16. Fehrmann RS, Karjalainen JM, Krajewska M et al (2015) Gene expression analysis identifies global gene dosage sensitivity in cancer. Nat Genet 47:115-125

17. Van Berkel A, Lenders JW, Timmers HJ (2014) Diagnosis of endocrine disease: biochemical diagnosis of phaeochromocytoma and paraganglioma. Eur J Endocrinol 170:R109-R119

18. Tharayil VS, Wouters MM, Stanich JE et al (2010) Lack of serotonin 5-HT2B receptor alters proliferation and network volume of interstitial cells of Cajal in vivo. Neurogastroenterol Motil 22: 462-469

19. Pore M, Meijer C, de Bock GH, Boersma-van Ek W, Terstappen LWMM, Groen HJM, Timens W, Kruyt FAE, Hiltermann TJN (2016) Cancer stem cells, epithelial to mesenchymal markers, and circulating tumor cells in small cell lung cancer. Clin Lung Cancer 17:535-542

20. Müller K, Gilbertz KP, Meineke V (2012) Serotonin and ionizing radiation synergistically affect proliferation and adhesion molecule expression of malignant melanoma cells. J Dermatol Sci 68:89-98

21. Henriksen R, Dizeyi N, Abrahamsson PA (2012) Expression of serotonin receptors 5-HT1A, 5-HT1B, 5-HT2B and 5-HT4 in ovary and ovarian tumours. Anticancer Res 32:1361-1366

22. Kopparapu PK, Tinzl M, Anagnostaki L, Persson JL, Dizeyi N (2013) Expression and localization of serotonin receptors in human breast cancer. Anticancer Res 33:363-370 
23. Saveanu A, Sebag F, Guillet B, Archange C, Essamet W, Barlier A, Palazzo FF, Taïeb D (2013) Targeting dopamine receptors subtype 2 (D2DR) in pheochromocytomas: head-to-head comparison between in vitro and in vivo findings. J Clin Endocrinol Metab 98: E1951-E1955

24. Saveanu A, Muresan M, De Micco C et al (2011) Expression of somatostatin receptors, dopamine D2 receptors, noradrenaline transporters, and vesicular monoamine transporters in 52 pheochromocytomas and paragangliomas. Endocr Relat Cancer 18:287-300

25. Stein MN, Bertino JR, Kaufman HL, Mayer T, Moss R, Silk A, Chan N, Malhotra J, Rodriguez L, Aisner J, Aiken RD, Haffty BG, DiPaola RS, Saunders T, Zloza A, Damare S, Beckett Y, Yu B, Najmi S, Gabel C, Dickerson S, Zheng L, el-Deiry WS, Allen JE, Stogniew M, Oster W, Mehnert JM (2017) First-in-human clinical trial of oral ONC201 in patients with refractory solid tumors. Clin Cancer Res 23:4163-4169
26. Arrillaga-Romany I, Chi AS, Allen JE, Oster W, Wen PY, Batchelor TT (2017) A phase 2 study of the imipridone ONC201, a selective DRD2 antagonist for oncology, administered every three weeks in recurrent glioblastoma. Oncotarget 8:79298-79304

27. Wang Y, Li J, Tohti M, Hu Y, Wang S, Li W, Lu Z, Ma C (2014) The expression profile of dopamine D2 receptor, MGMT and VEGF in different histological subtypes of pituitary adenomas: a study of 197 cases and indications for medical therapy. J Exp Clin Cancer Res 33:56

28. Hyman DM, Taylor BS, Baselga J (2017) Implementing genomedriven oncology. Cell 168:584-599

Publisher's Note Springer Nature remains neutral with regard to jurisdictional claims in published maps and institutional affiliations. 